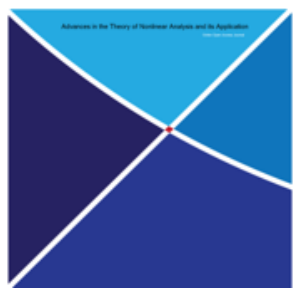

\title{
Periodic and asymptotically periodic solutions in nonlinear coupled Volterra integro-dynamic systems with infinite delay on time scales
}

\author{
Belkis Bordj ${ }^{1}$, Abdelouaheb Ardjouni ${ }^{1}$ \\ ${ }^{a}$ Department of Mathematics and Informatics, Souk Ahras University, Souk Ahras, Algeria.
}

\begin{abstract}
Let $\mathbb{T}$ be a periodic time scale. The purpose of this paper is to use Schauder's fixed point theorem to prove the existence of periodic and asymptotically periodic solutions of nonlinear coupled Volterra integro-dynamic systems with infinite delay on time scales. The results obtained here extend the work of Raffoul [22].

Keywords: Fixed points, Periodic solutions, Asymptotically periodic solutions, Volterra integro-dynamic systems, Time scales 2010 MSC: 34K13, 34K20, 45J05.
\end{abstract}

\section{Introduction}

Time scales calculus was initiated in 1988 by Stefan Hilger. It bridges the gap between continuous and discrete analysis and expands on both theories. Differential equations are defined on an interval of the set of real numbers while difference equations are defined on discrete sets. However, some physical systems are modeled by what is called dynamic equations because they are either differential equations, difference equations or a combination of both. This means that dynamic equations are defined on connected, discrete or combination of both types of sets. Hence, time scales calculus provides a generalization of differential and difference analysis, see [9, 10, 18, 20] and the references therein.

Delay dynamic equations arise from a variety of applications including in various fields of science and engineering such as applied sciences, practical problems concerning mechanics, the engineering technique

Email addresses: bordj.belkis2019@gmail.com (Belkis Bordj), abd_ardjouni@yahoo.fr (Abdelouaheb Ardjouni) 
fields, economy, control systems, physics, chemistry, biology, medicine, atomic energy, information theory, harmonic oscillator, nonlinear oscillations, conservative systems, stability and instability of geodesic on Riemannian manifolds, dynamics in Hamiltonian systems, etc, see [9, 10, 20, 25, 26]. In particular, problems concerning qualitative analysis of delay dynamic equations have received the attention of many authors, see [1]-[22], [24]-[26] and the references therein.

Let $\mathbb{T}$ be a periodic time scale such that $0 \in \mathbb{T}$. In this article, we are interested in the analysis of qualitative theory of periodic and asymptotically periodic solutions of coupled Volterra integro-dynamic equations. Inspired and motivated by the references in this paper, we consider the following nonlinear coupled Volterra integro-dynamic systems with infinite delay

$$
\left\{\begin{array}{c}
x^{\Delta}(t)=h_{1}(t) x(t)+h_{2}(t) y(t)+\int_{-\infty}^{t} a(t, s) f(x(s), y(s)) \Delta s, \\
y^{\Delta}(t)=p_{1}(t) y(t)+p_{2}(t) x(t)+\int_{-\infty}^{t} b(t, s) g(x(s), y(s)) \Delta s,
\end{array}\right.
$$

where $h_{1}, h_{2}, p_{1}, p_{2}, a$ and $b$ are rd-continuous functions, $f$ and $g$ are continuous functions. To show the existence of periodic and asymptotically periodic solutions of (1), we transform (1) into an integral system and then use Schauder's fixed point theorem. In the special case $\mathbb{T}=\mathbb{R}$, Raffoul in 22 show the existence of periodic and asymptotically periodic solutions of (1). Then, the results presented in this paper extend the main results in [22].

\section{Preliminaries}

A time scale is an arbitrary nonempty closed subset of real numbers. The study of dynamic equations on time scales is a fairly new subject, and research in this area is rapidly growing (see [1]-[13], [17]-[20] and papers therein). The theory of dynamic equations unifies the theories of differential equations and difference equations. We suppose that the reader is familiar with the basic concepts concerning the calculus on time scales for dynamic equations. Otherwise one can find in Bohner and Peterson books [9, 10, 20] most of the material needed to read this paper. We start by giving some definitions necessary for our work. The notion of periodic time scales is introduced in Kaufmann and Raffoul [19]. The following two definitions are borrowed from [19].

Definition 2.1. We say that a time scale $\mathbb{T}$ is periodic if there exists an $\omega>0$ such that if $t \in \mathbb{T}$ then $t \pm \omega \in \mathbb{T}$. For $\mathbb{T} \neq \mathbb{R}$, the smallest positive $\omega$ is called the period of the time scale.

Example 2.1. The following time scales are periodic.

1. $\mathbb{T}=\bigcup_{i=-\infty}^{\infty}[(2 i-1) h, 2 i h], h>0$ has period $\omega=2 h$.

2. $\mathbb{T}=h \mathbb{Z}$ has period $\omega=h$.

3. $\mathbb{T}=\mathbb{R}$.

4. $\mathbb{T}=\left\{t=k-q^{m}: k \in \mathbb{Z}, m \in \mathbb{N}_{0}\right\}$ where, $0<q<1$ has period $\omega=1$.

Remark 2.1 ([19]). All periodic time scales are unbounded above and below.

Definition 2.2. Let $\mathbb{T} \neq \mathbb{R}$ be a periodic time scale with period $\omega$. We say that the function $f: \mathbb{T} \rightarrow \mathbb{R}$ is periodic with period $T$ if there exists a natural number $n$ such that $T=n \omega, f(t \pm T)=f(t)$ for all $t \in \mathbb{T}$ and $T$ is the smallest number such that $f(t \pm T)=f(t)$.

If $\mathbb{T}=\mathbb{R}$, we say that $f$ is periodic with period $T>0$ if $T$ is the smallest positive number such that $f(t \pm T)=f(t)$ for all $t \in \mathbb{T}$.

Remark $2.2([19])$. If $\mathbb{T}$ is a periodic time scale with period $\omega$, then $\sigma(t \pm n \omega)=\sigma(t) \pm n \omega$. Consequently, the graininess function $\mu$ satisfies $\mu(t \pm n \omega)=\sigma(t \pm n \omega)-(t \pm n \omega)=\sigma(t)-t=\mu(t)$ and so, is a periodic function with period $\omega$. 
Definition $2.3([9])$. A function $f: \mathbb{T} \rightarrow \mathbb{R}$ is called $r d$-continuous provided it is continuous at every rightdense point $t \in \mathbb{T}$ and its left-sided limits exist, and is finite at every left-dense point $t \in \mathbb{T}$. The set of $r d$-continuous functions $f: \mathbb{T} \rightarrow \mathbb{R}$ will be denoted by

$$
C_{r d}=C_{r d}(\mathbb{T})=C_{r d}(\mathbb{T}, \mathbb{R}) .
$$

The set of functions $f: \mathbb{T} \rightarrow \mathbb{R}$ that are differentiable and whose derivative is rd-continuous is denoted by

$$
C_{r d}^{1}=C_{r d}^{1}(\mathbb{T})=C_{r d}^{1}(\mathbb{T}, \mathbb{R}) .
$$

Definition $2.4([9])$. For $f: \mathbb{T} \rightarrow \mathbb{R}$, we define $f^{\Delta}(t)$ to be the number (if it exists) with the property that for any given $\varepsilon>0$, there exists a neighborhood $U$ of $t$ such that

$$
\left|(f(\sigma(t))-f(s))-f^{\Delta}(t)(\sigma(t)-s)\right|<\varepsilon|\sigma(t)-s| \text { for all } s \in U .
$$

The function $f^{\Delta}: \mathbb{T}^{k} \rightarrow \mathbb{R}$ is called the delta (or Hilger) derivative of $f$ on $\mathbb{T}^{k}$.

Definition $2.5([9])$. A function $p: \mathbb{T} \rightarrow \mathbb{R}$ is called regressive provided $1+\mu(t) p(t) \neq 0$ for all $t \in \mathbb{T}$. The set of all regressive and $r d$-continuous functions $p: \mathbb{T} \rightarrow \mathbb{R}$ will be denoted by $\mathcal{R}=\mathcal{R}(\mathbb{T}, \mathbb{R})$. We define the set $\mathcal{R}^{+}$of all positively regressive elements of $\mathcal{R}$ by

$$
\mathcal{R}^{+}=\mathcal{R}^{+}(\mathbb{T}, \mathbb{R})=\{p \in \mathcal{R}: 1+\mu(t) p(t)>0, \forall t \in \mathbb{T}\} .
$$

Definition $2.6([9])$. Let $p \in \mathcal{R}$, then the generalized exponential function $e_{p}$ is defined as the unique solution of the initial value problem

$$
x^{\Delta}(t)=p(t) x(t), x(s)=1, \text { where } s \in \mathbb{T} .
$$

An explicit formula for $e_{p}(t, s)$ is given by

$$
e_{p}(t, s)=\exp \left(\int_{s}^{t} \xi_{\mu(v)}(p(v)) \Delta v\right), \text { for all } s, t \in \mathbb{T},
$$

with

$$
\xi_{\mu}(p)= \begin{cases}\frac{\log (1+\mu p)}{\mu} & \text { if } \mu \neq 0, \\ p & \text { if } \mu=0,\end{cases}
$$

where $\log$ is the principal logarithm function.

Lemma 2.1 ([9]). Let $p, q \in \mathcal{R}$. Then

(i) $e_{0}(t, s) \equiv 1$ and $e_{p}(t, t) \equiv 1$,

(ii) $e_{p}(\sigma(t), s)=(1+\mu(t) p(t)) e_{p}(t, s)$,

(iii) $\frac{1}{e_{p}(t, s)}=e_{\ominus p}(t, s)$ where, $\ominus p(t)=-\frac{p(t)}{1+\mu(t) p(t)}$,

(iv) $e_{p}(t, s)=\frac{1}{e_{p}(s, t)}=e_{\ominus p}(s, t)$

(v) $e_{p}(t, s) e_{p}(s, r)=e_{p}(t, r)$,

(vi) $\left(\frac{1}{e_{p}(\cdot, s)}\right)^{\Delta}=-\frac{p(t)}{e_{p}^{\sigma}(\cdot, s)}$.

Lemma 2.2 ([1]). If $p \in \mathcal{R}^{+}$, then

$$
0<e_{p}(t, s) \leq \exp \left(\int_{s}^{t} p(v) \Delta v\right), \forall t \in \mathbb{T} .
$$


The proof of the main results in the next section is based upon an application of the following Schauder fixed point theorem.

Theorem 2.1 (Schauder's fixed point theorem [23]). Let $X$ be a Banach space, and $\Omega$ be a convex closed bounded subset of $X$. If $E: \Omega \rightarrow \Omega$ is completely continuous, then $E$ has at least one fixed point in $\Omega$.

Definition 2.7. A map is completely continuous if it is continuous and it maps bounded sets into relatively compact sets.

\section{Periodic solutions}

Let $T>0, T \in \mathbb{T}$ be fixed and if $\mathbb{T} \neq \mathbb{R}, T=n \omega$ for some $n \in \mathbb{N}$. By the notation $[a, b]$ we mean

$$
[a, b]=\{t \in \mathbb{T}: a \leq t \leq b\},
$$

unless otherwise specified. The intervals $[a, b),(a, b]$ and $(a, b)$ are defined similarly. Define

$$
P_{T}=\left\{(\phi, \psi) \in C_{r d}\left(\mathbb{T}, \mathbb{R}^{2}\right):(\phi, \psi)(t+T)=(\phi, \psi)(t)\right\},
$$

where both $\varphi$ and $\psi$ are real valued rd-continuous functions on $\mathbb{T}$. Then $P_{T}$ is a Banach space when endowed with the maximum norm

$$
\|(x, y)\|=\max \left\{\max _{t \in[0, T]}|x(t)|, \max _{t \in[0, T]}|y(t)|\right\},
$$

see [3]. Throughout this paper, we assume that $h_{1}, p_{1} \in \mathcal{R}^{+}, h_{1}, p_{1}, h_{2}, p_{2}, a$ and $b$ are rd-continuous functions, $f$ and $g$ are continuous functions, and

$$
\begin{aligned}
a(t+T, s+T) & =a(t, s), b(t+T, s+T)=b(t, s), \\
p_{i}(t+T) & =p_{i}(t), h_{i}(t+T)=h_{i}(t), i=1,2,
\end{aligned}
$$

for all $t \in \mathbb{T}$. Also, we assume that

$$
e_{h_{1}}(T, 0) \neq 1, e_{p_{1}}(T, 0) \neq 1 .
$$

The following lemma is fundamental to our results.

Lemma 3.1. Assume (2) and (3) hold. If $(x, y) \in P_{T}$, then $(x, y)$ is a solution of (1) if and only if

$$
\begin{aligned}
x(t) & =\int_{t}^{t+T} \frac{e_{h_{1}}(t+T, \sigma(u))}{1-e_{h_{1}}(t+T, t)} h_{2}(u) y(u) \Delta u \\
& +\int_{t}^{t+T} \frac{e_{h_{1}}(t+T, \sigma(u))}{1-e_{h_{1}}(t+T, t)} \int_{-\infty}^{u} a(u, s) f(x(s), y(s)) \Delta s \Delta u,
\end{aligned}
$$

and

$$
\begin{aligned}
y(t) & =\int_{t}^{t+T} \frac{e_{p_{1}}(t+T, \sigma(u))}{1-e_{p_{1}}(t+T, t)} p_{2}(u) x(u) \Delta u \\
& +\int_{t}^{t+T} \frac{e_{p_{1}}(t+T, \sigma(u))}{1-e_{p_{1}}(t+T, t)} \int_{-\infty}^{u} b(u, s) g(x(s), y(s)) \Delta s \Delta u .
\end{aligned}
$$

Proof. Let $(x, y) \in P_{T}$ be a solution of (1). First we write the first equation of (1) as

$$
x^{\Delta}(t)-h_{1}(t) x(t)=h_{2}(t) y(t)+\int_{-\infty}^{t} a(t, s) f(x(s), y(s)) \Delta s
$$


Multiplying both side by $e_{\ominus h_{1}}(\sigma(t), 0)$ and then integrate from $t$ to $t+T$ to obtain

$$
\begin{aligned}
& \int_{t}^{t+T}\left[x(s) e_{\ominus h_{1}}(s, 0)\right]^{\Delta} \Delta s \\
& =\int_{t}^{t+T} e_{\ominus h_{1}}(\sigma(u), 0) h_{2}(u) y(u) \Delta u \\
& +\int_{t}^{t+T} e_{\ominus h_{1}}(\sigma(u), 0) \int_{-\infty}^{u} a(u, s) f(x(s), y(s)) \Delta s \Delta u,
\end{aligned}
$$

then

$$
\begin{aligned}
& x(t+T) e_{\ominus h_{1}}(t+T, 0)-x(t) e_{\ominus h_{1}}(t, 0) \\
& =\int_{t}^{t+T} e_{\ominus h_{1}}(\sigma(u), 0) h_{2}(u) y(u) \Delta u \\
& +\int_{t}^{t+T} e_{\ominus h_{1}}(\sigma(u), 0) \int_{-\infty}^{u} a(u, s) f(x(s), y(s)) \Delta s \Delta u .
\end{aligned}
$$

Periodicity of $x$ gives

$$
\begin{aligned}
& x(t)\left(1-e_{h_{1}}(t+T, t)\right) \\
& =\int_{t}^{t+T} h_{2}(u) y(u) e_{h_{1}}(t+T, \sigma(u)) \Delta u \\
& +\int_{t}^{t+T} e_{h_{1}}(t+T, \sigma(u)) \int_{-\infty}^{u} a(u, s) f(x(s), y(s)) \Delta s \Delta u .
\end{aligned}
$$

Thus

$$
\begin{aligned}
x(t) & =\int_{t}^{t+T} \frac{e_{h_{1}}(t+T, \sigma(u))}{1-e_{h_{1}}(t+T, t)} h_{2}(u) y(u) \Delta u \\
& +\int_{t}^{t+T} \frac{e_{h_{1}}(t+T, \sigma(u))}{\left(1-e_{h_{1}}(t+T, t)\right)} \int_{-\infty}^{u} a(u, s) f(x(s), y(s)) \Delta s \Delta u .
\end{aligned}
$$

In the similar fashion

$$
\begin{aligned}
y(t) & =\int_{t}^{t+T} \frac{e_{p_{1}}(t+T, \sigma(u))}{1-e_{p_{1}}(t+T, t)} p_{2}(u) x(u) \Delta u \\
& +\int_{t}^{t+T} \frac{e_{p_{1}}(t+T, \sigma(u))}{1-e_{p_{1}}(t+T, t)} \int_{-\infty}^{u} b(u, s) g(x(s), y(s)) \Delta s \Delta u .
\end{aligned}
$$

The proof is complete by reversing every step.

Since $h_{1}, h_{2}, p_{1}$ and $p_{2}$ are rd-continuous $T$-periodic functions, then there exist positive constants $H_{1}$, $H_{2}, P_{1}$ and $P_{2}$ such that $\left|h_{i}(t)\right| \leq H_{i}$ and $\left|p_{i}(t)\right| \leq P_{i}$ for $i=1,2$. Let $L_{1}$ and $L_{2}$ be positive constants such that $0<L_{1} H_{2} T<1$ and $0<L_{2} P_{2} T<1$. Also, assume there exist positive constants $M_{1}, M_{2}, A$ and $B$ such that

$$
\begin{gathered}
|f(x, y)| \leq M_{1}, \\
|g(x, y)| \leq M_{2}, \\
\left|\frac{e_{h_{1}}(t+T, \sigma(u))}{1-e_{h_{1}}(t+T, t)}\right| \leq L_{1}, \\
\left|\frac{e_{p_{1}}(t+T, \sigma(u))}{1-e_{p_{1}}(t+T, t)}\right| \leq L_{2},
\end{gathered}
$$




$$
\int_{-\infty}^{u}|a(u, s)| \Delta s \leq A
$$

and

$$
\int_{-\infty}^{u}|b(u, s)| \Delta s \leq B
$$

Set

$$
M=\max \left\{\frac{L_{1} A M_{1} T}{1-L_{1} H_{2} T}, \frac{L_{2} B M_{2} T}{1-L_{2} P_{2} T}\right\} .
$$

We define a subset $\Omega_{x, y}$ of $P_{T}$ as follows

$$
\Omega_{x, y}=\left\{(x, y) \in P_{T}:\|(x, y)\| \leq M\right\} .
$$

Then $\Omega_{x, y}$ is a bounded closed convex subset of $P_{T}$. Now for $(x, y) \in \Omega_{x, y}$ we can define an operator $E: \Omega_{x, y} \rightarrow P_{T}$ by

$$
E(x, y)(t)=\left(E_{1}(x, y)(t), E_{2}(x, y)(t)\right)
$$

where

$$
\begin{aligned}
E_{1}(x, y)(t) & =\int_{t}^{t+T} \frac{e_{h_{1}}(t+T, \sigma(u))}{1-e_{h_{1}}(t+T, t)} h_{2}(u) y(u) \Delta u \\
& +\int_{t}^{t+T} \frac{e_{h_{1}}(t+T, \sigma(u))}{1-e_{h_{1}}(t+T, t)} \int_{-\infty}^{u} a(u, s) f(x(s), y(s)) \Delta s \Delta u
\end{aligned}
$$

and

$$
\begin{aligned}
E_{2}(x, y)(t) & =\int_{t}^{t+T} \frac{e_{p_{1}}(t+T, \sigma(u))}{1-e_{p_{1}}(t+T, t)} p_{2}(u) x(u) \Delta u \\
& +\int_{t}^{t+T} \frac{e_{p_{1}}(t+T, \sigma(u))}{1-e_{p_{1}}(t+T, t)} \int_{-\infty}^{u} b(u, s) g(x(s), y(s)) \Delta s \Delta u .
\end{aligned}
$$

Theorem 3.1. Suppose (2), (3) and (6)-(11) hold. Then (1) has a T-periodic solution.

Proof. It is clear from Lemma 3.1 that $E_{1}(x, y)(t+T)=E_{1}(x, y)(t)$ and $E_{2}(x, y)(t+T)=E_{2}(x, y)(t)$. Therefore, $E(x, y)(t+T)=E(x, y)(t)$. Moreover, if $(x, y) \in \Omega_{x, y}$, then

$$
\begin{aligned}
\left|E_{1}(x, y)(t)\right| & \leq \int_{t}^{t+T}\left|\frac{e_{h_{1}}(t+T, \sigma(u))}{1-e_{h_{1}}(t+T, t)}\right|\left|h_{2}(u)\right||y(u)| \Delta u \\
& +\int_{t}^{t+T}\left|\frac{e_{h_{1}}(t+T, \sigma(u))}{1-e_{h_{1}}(t+T, t)}\right| \int_{-\infty}^{u}|a(u, s)||f(x(s), y(s))| \Delta s \Delta u \\
& \leq L_{1} H_{2} T M+L_{1} A M_{1} T .
\end{aligned}
$$

Since

$$
\frac{L_{1} A M_{1} T}{1-L_{1} H_{2} T} \leq M
$$

we have

$$
\left|E_{1}(x, y)(t)\right| \leq L_{1} H_{2} T M+M\left(1-L_{1} H_{2} T\right)=M .
$$

In the same manner we can see that

$$
\left|E_{2}(x, y)(t)\right| \leq M
$$

So, we have

$$
|E(x, y)(t)| \leq M
$$


Thus, $E$ maps $\Omega_{x, y}$ into itself, i.e $E\left(\Omega_{x, y}\right) \subseteq \Omega_{x, y}$. Now, we shall prove that $E$ is continuous. Let $\left\{\left(x^{l}, y^{l}\right)\right\}$ be a sequence in $\Omega_{x, y}$ such that

$$
\lim _{l \rightarrow \infty}\left\|\left(x^{l}, y^{l}\right)-(x, y)\right\|=0 .
$$

Since $\Omega_{x, y}$ is closed, we have $(x, y) \in \Omega_{x, y}$. Then by definition of $E$ we have

$$
\begin{aligned}
& \left\|E\left(x^{l}, y^{l}\right)-E(x, y)\right\| \\
& =\max \left\{\max _{t \in[0, T]}\left|E_{1}\left(x^{l}, y^{l}\right)-E_{1}(x, y)\right|, \max _{t \in[0, T]}\left|E_{2}\left(x^{l}, y^{l}\right)-E_{2}(x, y)\right|\right\},
\end{aligned}
$$

in which

$$
\begin{aligned}
& \left|E_{1}\left(x^{l}, y^{l}\right)-E_{1}(x, y)\right| \\
& =\mid \int_{t}^{t+T} \frac{e_{h_{1}}(t+T, \sigma(u))}{1-e_{h_{1}}(t+T, t)} h_{2}(u) y^{l}(u) \Delta u \\
& +\int_{t}^{t+T} \frac{e_{h_{1}}(t+T, \sigma(u))}{1-e_{h_{1}}(t+T, t)} \int_{-\infty}^{u} a(u, s) f\left(x^{l}(s), y^{l}(s)\right) \Delta s \Delta u \\
& -\int_{t}^{t+T} \frac{e_{h_{1}}(t+T, \sigma(u))}{1-e_{h_{1}}(t+T, t)} h_{2}(u) y(u) \Delta u \\
& -\int_{t}^{t+T} \frac{e_{h_{1}}(t+T, \sigma(u))}{1-e_{h_{1}}(t+T, t)} \int_{-\infty}^{u} a(u, s) f(x(s), y(s)) \Delta s \Delta u \mid \\
& \leq \int_{t}^{t+T}\left|\frac{e_{h_{1}}(t+T, \sigma(u))}{1-e_{h_{1}}(t+T, t)}\right| h_{2}(u)|| y^{l}(u)-y(u) \mid \Delta u \\
& +\int_{t}^{t+T}\left|\frac{e_{h_{1}}(t+T, \sigma(u))}{1-e_{h_{1}}(t+T, t)}\right| \int_{-\infty}^{u}|a(u, s)| f\left(x^{l}(s), y^{l}(s)\right)-f(x(s), y(s)) \mid \Delta s \Delta u
\end{aligned}
$$

The continuity of $f$ along with the Lebesgue dominated convergence theorem implies that

$$
\lim _{l \rightarrow \infty} \max _{t \in[0, T]}\left|E_{1}\left(x^{l}, y^{l}\right)(t)-E_{1}(x, y)(t)\right|=0 .
$$

In similar way we have

$$
\lim _{l \rightarrow \infty} \max _{t \in[0, T]}\left|E_{2}\left(x^{l}, y^{l}\right)(t)-E_{2}(x, y)(t)\right|=0
$$

Thus,

$$
\lim _{l \rightarrow \infty}\left\|E\left(x^{l}, y^{l}\right)-E(x, y)\right\|=0 .
$$

This show that $E$ is a continuous map. To show that the map $E$ is completely continuous, we will show that $E\left(\Omega_{x, y}\right)$ is relatively compact. We know that $E\left(\Omega_{x, y}\right) \subseteq \Omega_{x, y}$, which means $E\left(\Omega_{x, y}\right)$ is uniformly bounded because $\Omega_{x, y}$ is uniformly bounded. Moreover, a direct calculation shows that

$$
E_{1}(x, y)^{\triangle}(t)=h_{1}(t) E_{1}(x, y)(t)+h_{2}(t) y(t)+\int_{-\infty}^{t} a(t, s) f(x(s), y(s)) \Delta s,
$$

and

$$
E_{2}(x, y)^{\triangle}(t)=p_{1}(t) x(t)+p_{2}(t) E_{2}(x, y)(t)+\int_{-\infty}^{t} b(t, s) g(x(s), y(s)) \Delta s .
$$

Then, there exists a positive constant $L$ such that

$$
\left|E_{1}(x, y)^{\triangle}(t)\right| \leq H_{1} M+H_{2} M+M_{1} A \leq L,
$$


and

$$
\left|E_{2}(x, y)^{\triangle}(t)\right| \leq P_{1} M+P_{2} M+M_{2} B \leq L .
$$

This means that $\left|E(x, y)^{\triangle}(t)\right| \leq L$. Therefore the set $E\left(\Omega_{x, y}\right)$ is equicontinuous, and hence by ArzelaAscoli's theorem, it is relatively compact. By Schauder's fixed point theorem, we conclude that there exists $(x, y) \in \Omega_{x, y}$ such that $(x, y)=E(x, y)$.

Now, we relax condition (7).

Theorem 3.2. Suppose (2), (3), (6) and (9)-(11) hold. In addition, we assume the existence of continuous nondecreasing function $G$ such that

$$
|g(x, y)| \leq g(|x|, y) \leq Q G(|x|) \text { for some positive constant } Q,
$$

and for $u>0$ we ask that

$$
\frac{G(u)}{u} \leq \frac{1-L_{2} P_{2} T}{L_{2} B Q T}
$$

Then (1) has a T-periodic solution.

Proof. Set

$$
M=\max \left\{\frac{L_{1} A M_{1} T}{1-L_{1} H_{2} T}, \frac{L_{2} B Q G(M) T}{1-L_{2} P_{2} T}\right\} .
$$

For $(x, y) \in \Omega_{x, y}$, we have from the proof of Theorem 3.1 that

$$
\left|E_{1}(x, y)(t)\right| \leq M \text {. }
$$

Thus,

$$
\begin{aligned}
\left|E_{2}(x, y)(t)\right| & \leq \int_{t}^{t+T}\left|\frac{e_{p_{1}}(t+T, \sigma(u))}{1-e_{p_{1}}(t+T, t)}\right|\left|p_{2}(u)\right||x(u)| \Delta u \\
& +\int_{t}^{t+T}\left|\frac{e_{p_{1}}(t+T, \sigma(u))}{1-e_{p_{1}}(t+T, t)}\right| \int_{-\infty}^{u}|b(u, s)||g(x(s), y(s))| \Delta s \Delta u \\
& \leq \int_{t}^{t+T}\left|\frac{e_{p_{1}}(t+T, \sigma(u))}{1-e_{p_{1}}(t+T, t)}\right|\left|p_{2}(u)\right|\left|E_{1}(x, y)(u)\right| \Delta u \\
& +\int_{t}^{t+T}\left|\frac{e_{p_{1}}(t+T, \sigma(u))}{1-e_{p_{1}}(t+T, t)}\right| \int_{-\infty}^{u}|b(u, s)|\left|g\left(\left|E_{1}(x, y)(s)\right|, y(s)\right)\right| \Delta s \Delta u \\
& \leq \int_{t}^{t+T}\left|\frac{e_{p_{1}}(t+T, \sigma(u))}{1-e_{p_{1}}(t+T, t)}\right|\left|p_{2}(u)\right|\left|E_{1}(x, y)(u)\right| \Delta u \\
& +Q \int_{t}^{t+T}\left|\frac{e_{p_{1}}(t+T, \sigma(u))}{1-e_{p_{1}}(t+T, t)}\right| \int_{-\infty}^{u}|b(u, s)| G\left(\left|E_{1}(x, y)(s)\right|\right) \Delta s \Delta u \\
& \leq M \int_{t}^{t+T}\left|\frac{e_{p_{1}}(t+T, \sigma(u))}{1-e_{p_{1}}(t+T, t)}\right|\left|p_{2}(u)\right| \Delta u \\
& +Q \int_{t}^{t+T}\left|\frac{e_{p_{1}}(t+T, \sigma(u))}{1-e_{p_{1}}(t+T, t)}\right| \int_{-\infty}^{u}|b(u, s)| G(M) \Delta s \Delta u \\
& \leq M L_{2} P_{2} T+L_{2} B Q G(M) T \frac{M\left(1-L_{2} P_{2} T\right)}{L_{2} B Q G(M) T}=M .
\end{aligned}
$$

The rest of the proof follows along the lines of the proof of Theorem 3.1 .

In the next theorem we relax condition (6). 
Theorem 3.3. Suppose (2), (3), and (7)-(11) hold. In addition, we assume the existence of continuous nondecreasing function $W$ such that

$$
|f(x, y)| \leq f(x,|y|) \leq R W(|y|) \text { for some positive constant } R,
$$

and for $u>0$ we ask that

$$
\frac{W(u)}{u} \leq \frac{1-L_{1} H_{2} T}{L_{1} A R T}
$$

Then (1) has a T-periodic solution.

Proof. Set

$$
M=\max \left\{\frac{L_{1} A R W(M) T}{1-L_{1} H_{2} T}, \frac{L_{2} B M_{2} T}{1-L_{2} P_{2} T}\right\} .
$$

For $(x, y) \in \Omega_{x, y}$, we have from the proof of Theorem 3.1 that

$$
\left|E_{2}(x, y)(t)\right| \leq M \text {. }
$$

Thus,

$$
\begin{aligned}
\left|E_{1}(x, y)(t)\right| & \leq \int_{t}^{t+T}\left|\frac{e_{h_{1}}(t+T, \sigma(u))}{1-e_{h_{1}}(t+T, t)}\right|\left|h_{2}(u)\right||y(u)| \Delta u \\
& +\int_{t}^{t+T}\left|\frac{e_{h_{1}}(t+T, \sigma(u))}{1-e_{h_{1}}(t+T, t)}\right| \int_{-\infty}^{u}|a(u, s)||f(x(s), y(s))| \Delta s \Delta u \\
& \leq \int_{t}^{t+T}\left|\frac{e_{h_{1}}(t+T, \sigma(u))}{1-e_{h_{1}}(t+T, t)}\right|\left|h_{2}(u)\right|\left|E_{2}(x, y)(u)\right| \Delta u \\
& +\int_{t}^{t+T}\left|\frac{e_{h_{1}}(t+T, \sigma(u))}{1-e_{h_{1}}(t+T, t)}\right| \int_{-\infty}^{u}|a(u, s)|\left|f\left(x(s), E_{2}(x, y)(s)\right)\right| \Delta s \Delta u \\
& \leq \int_{t}^{t+T}\left|\frac{e_{h_{1}}(t+T, \sigma(u))}{1-e_{h_{1}}(t+T, t)}\right|\left|h_{2}(u)\right|\left|E_{2}(x, y)(u)\right| \Delta u \\
& +R \int_{t}^{t+T}\left|\frac{e_{h_{1}}(t+T, \sigma(u))}{1-e_{h_{1}}(t+T, t)}\right| \int_{-\infty}^{u}|a(u, s)| W\left(\left|E_{2}(x, y)(s)\right|\right) \Delta s \Delta u \\
& \leq M \int_{t}^{t+T}\left|\frac{e_{h_{1}}(t+T, \sigma(u))}{1-e_{h_{1}}(t+T, t)}\right|\left|h_{2}(u)\right| \Delta u \\
& +R \int_{t}^{t+T}\left|\frac{e_{h_{1}}(t+T, \sigma(u))}{1-e_{h_{1}}(t+T, t)}\right| \int_{-\infty}^{u}|a(u, s)| W(M) \Delta s \Delta u \\
& \leq M L_{1} H_{2} T+L_{1} A R W(M) \\
& M \frac{M\left(1-L_{1} H_{2} T\right)}{L_{1} A R W(M) T}=M .
\end{aligned}
$$

The rest of the proof follows along the lines of the proof of Theorem 3.1 .

\section{Asymptotically periodic solutions}

In this section, we obtain asymptotically periodic solutions of (1).

Definition 4.1. A function $x$ is called asymptotically T-periodic if there exist two functions $x_{1}$ and $x_{2}$ such that $x_{1}$ is T-periodic, $\lim _{t \rightarrow \infty} x_{2}(t)=0$ and $x(t)=x_{1}(t)+x_{2}(t)$ for all $t \in \mathbb{T}$.

In this section we do not assume the periodicity condition on the functions $h_{2}, p_{2}, a$ and $b$. We only assume $h_{1}, p_{1} \in \mathcal{R}^{+}, h_{1}$ and $p_{1}$ are $T$-periodic, and

$$
e_{h_{1}}(T, 0)=e_{p_{1}}(T, 0)=1 .
$$


Since $h_{1}$ and $p_{1}$ are $T$-periodic, there are constants $S_{k}, s_{k}, M_{k}^{*}, m_{k}, k=1,2$, such that

$$
\begin{aligned}
m_{1} & \leq e_{h_{1}}(t, 0) \leq M_{1}^{*} \text { and } m_{2} \leq e_{p_{1}}(t, 0) \leq M_{2}^{*}, \\
s_{1} & \leq e_{\ominus h_{1}}(\sigma(t), 0) \leq S_{1} \text { and } s_{2} \leq e_{\ominus p_{1}}(\sigma(t), 0) \leq S_{2} .
\end{aligned}
$$

Also, we assume that there are positive constants $A^{*}, B^{*}, M_{3}^{*}$ and $M_{4}^{*}$ such that

$$
\int_{0}^{\infty} \int_{-\infty}^{u}|a(u, s)| \Delta s \Delta u \leq A^{*} \text { and } \int_{0}^{\infty} \int_{-\infty}^{u}|b(u, s)| \Delta s \Delta u \leq B^{*}
$$

and

$$
\int_{0}^{\infty}\left|h_{2}(u)\right| \Delta u \leq M_{3}^{*} \text { and } \int_{0}^{\infty}\left|p_{2}(u)\right| \Delta u \leq M_{4}^{*} .
$$

In addition, we suppose that

$$
\lim _{t \rightarrow \infty} \int_{t}^{\infty} \int_{-\infty}^{u}|a(u, s)| \Delta s \Delta u=\lim _{t \rightarrow \infty} \int_{t}^{\infty} \int_{-\infty}^{u}|b(u, s)| \Delta s \Delta u=0,
$$

and

$$
\lim _{t \rightarrow \infty} \int_{t}^{\infty}\left|h_{2}(u)\right| \Delta u=\lim _{t \rightarrow \infty} \int_{t}^{\infty}\left|p_{2}(u)\right| \Delta u=0 .
$$

Finally, we Assume that

$$
1-m_{1}^{-1} S_{1} M_{3}^{*}>0 \text { and } 1-m_{2}^{-1} S_{2} M_{4}^{*}>0 .
$$

Theorem 4.1. Suppose that (6), (7) and (15)-(21) hold. Then the system (1) has asymptotically T-periodic solution $(x, y)$ satisfying

$$
\begin{aligned}
& x(t)=x_{1}(t)+x_{2}(t), \\
& y(t)=y_{1}(t)+y_{2}(t),
\end{aligned}
$$

where

$$
x_{1}(t)=c_{1} e_{h_{1}}(t, 0), y_{1}(t)=c_{2} e_{p_{1}}(t, 0), c_{1}, c_{2} \in \mathbb{R}^{*},
$$

and

$$
\lim _{t \rightarrow \infty} x_{2}(t)=\lim _{t \rightarrow \infty} y_{2}(t)=0
$$

Proof. Define

$$
\begin{aligned}
P_{T}^{*}= & \left\{(\varphi, \psi) \in C_{r d}\left(\mathbb{T}, \mathbb{R}^{2}\right): \varphi=\varphi_{1}+\varphi_{2}, \psi=\psi_{1}+\psi_{2},\right. \\
& \left.\left(\varphi_{1}, \psi_{1}\right)(T+t)=\left(\varphi_{1}, \psi_{1}\right)(t),\left(\varphi_{2}, \psi_{2}\right)(t) \rightarrow(0,0) \text { as } t \rightarrow \infty\right\} .
\end{aligned}
$$

Then $P_{T}^{*}$ is a Banach space when endowed with the maximum norm

$$
\|(x, y)\|=\max \left\{\max _{t \in[0, T]}|x(t)|, \max _{t \in[0, T]}|y(t)|\right\} .
$$

Define a subset $\Omega_{x, y}$ of $P_{T}^{*}$ as follows. For a positive constant $W^{*}$ to be defined later in the proof, let

$$
\Omega_{x, y}=\left\{(x, y) \in P_{T}^{*}:\|(x, y)\| \leq W^{*}\right\} .
$$

Then $\Omega_{x, y}$ is a bounded closed convex subset of $P_{T}^{*}$. Now, for $(x, y) \in \Omega_{x, y}$ we can define an operator $F: \Omega_{x, y} \rightarrow P_{T}^{*}$ by

$$
F(x, y)(t)=\left(F_{1}(x, y)(t), F_{2}(x, y)(t)\right)
$$


where

$$
\begin{aligned}
F_{1}(x, y)(t) & =c_{1} e_{h_{1}}(t, 0)-\int_{t}^{\infty} e_{\ominus h_{1}}(\sigma(u), t) h_{2}(u) y(u) \Delta u \\
& -\int_{t}^{\infty} e_{\ominus h_{1}}(\sigma(u), t) \int_{-\infty}^{u} a(u, s) f(x(s), y(s)) \Delta s \Delta u
\end{aligned}
$$

and

$$
\begin{aligned}
F_{2}(x, y)(t) & =c_{2} e_{p_{1}}(t, 0)-\int_{t}^{\infty} e_{\ominus p_{1}}(\sigma(u), t) p_{2}(u) x(u) \Delta u \\
& -\int_{t}^{\infty} e_{\ominus p_{1}}(\sigma(u), t) \int_{-\infty}^{u} b(u, s) g(x(s), y(s)) \Delta s \Delta u .
\end{aligned}
$$

We will show that the mapping $F$ has a fixed point in $\Omega_{x, y}$. Set

$$
W^{*}=\max \left\{\frac{c_{1} M_{1}^{*}+m_{1}^{-1} S_{1} M_{1} A^{*}}{1-m_{1}^{-1} S_{1} M_{3}^{*}}, \frac{c_{2} M_{2}^{*}+m_{2}^{-1} S_{2} M_{2} B^{*}}{1-m_{2}^{-1} S_{2} M_{4}^{*}}\right\} .
$$

We note that $W^{*}$ is well defined due to 21 . First, we demonstrate that $F\left(\Omega_{x, y}\right) \subseteq \Omega_{x, y}$. If $(x, y) \in \Omega_{x, y}$, then

$$
\begin{aligned}
\left|F_{1}(x, y)(t)\right| & \leq c_{1} e_{h_{1}}(t, 0)+\int_{t}^{\infty}\left|e_{\ominus h_{1}}(\sigma(u), t)\right|\left|h_{2}(u)\right||y(u)| \Delta u \\
& +\int_{t}^{\infty}\left|e_{\ominus h_{1}}(\sigma(u), t)\right| \int_{-\infty}^{u}|a(u, s)||f(x(s), y(s))| \Delta s \Delta u \\
& \leq c_{1} M_{1}^{*}+m_{1}^{-1} S_{1} W^{*} \int_{0}^{\infty}\left|h_{2}(u)\right| \Delta u \\
& +m_{1}^{-1} S_{1} M_{1} \int_{0}^{\infty} \int_{-\infty}^{u}|a(u, s)| \Delta s \Delta u \\
& \leq c_{1} M_{1}^{*}+m_{1}^{-1} S_{1} W^{*} M_{3}^{*}+m_{1}^{-1} S_{1} M_{1} A^{*} .
\end{aligned}
$$

In similar way we have

$$
\left|F_{2}(x, y)(t)\right| \leq c_{2} M_{2}^{*}+m_{2}^{-1} S_{2} W^{*} M_{4}^{*}+m_{2}^{-1} S_{2} M_{2} B^{*}
$$

This implies that

$$
\left|F_{1}(x, y)(t)\right| \leq c_{1} M_{1}^{*}+m_{1}^{-1} S_{1} W^{*} M_{3}^{*}+m_{1}^{-1} S_{1} M_{1} A \leq W^{*},
$$

and

$$
\left|F_{2}(x, y)(t)\right| \leq c_{2} M_{2}^{*}+m_{2}^{-1} S_{2} W^{*} M_{4}^{*}+m_{2}^{-1} S_{2} M_{2} B \leq W^{*}
$$

Thus,

$$
\|F(x, y)\| \leq W^{*}
$$

By letting

$$
\begin{aligned}
x_{2}(t) & =-\int_{t}^{\infty} e_{\ominus h_{1}}(\sigma(u), t) h_{2}(u) y(u) \Delta u \\
& -\int_{t}^{\infty} e_{\ominus h_{1}}(\sigma(u), t) \int_{-\infty}^{u} a(u, s) f(x(s), y(s)) \Delta s \Delta u,
\end{aligned}
$$

and

$$
\begin{aligned}
y_{2}(t) & =-\int_{t}^{\infty} e_{\ominus p_{1}}(\sigma(u), t) p_{2}(u) x(u) \Delta u \\
& -\int_{t}^{\infty} e_{\ominus p_{1}}(\sigma(u), t) \int_{-\infty}^{u} b(u, s) g(x(s), y(s)) \Delta s \Delta u .
\end{aligned}
$$


Then,

$$
\begin{aligned}
\lim _{t \rightarrow \infty}\left|x_{2}(t)\right| \leq & m_{1}^{-1} S_{1} W^{*} \lim _{t \rightarrow \infty} \int_{t}^{\infty}\left|h_{2}(u)\right| \Delta u \\
& +m_{1}^{-1} S_{1} M_{1} \lim _{t \rightarrow \infty} \int_{t}^{\infty} \int_{-\infty}^{u}|a(u, s)| \Delta s \Delta u=0,
\end{aligned}
$$

and

$$
\begin{aligned}
& \lim _{t \rightarrow \infty}\left|y_{2}(t)\right| \leq m_{2}^{-1} S_{2} W^{*} \lim _{t \rightarrow \infty} \int_{t}^{\infty}\left|p_{2}(u)\right| \Delta u \\
&+m_{2}^{-1} S_{2} M_{2} \lim _{t \rightarrow \infty} \int_{t}^{\infty} \int_{-\infty}^{u}|b(u, s)| \Delta s \Delta u=0 .
\end{aligned}
$$

Hence,

$$
\lim _{t \rightarrow \infty} x_{2}(t)=0 \text { and } \lim _{t \rightarrow \infty} y_{2}(t)=0 .
$$

We will prove that $x_{1}$ and $y_{1}$ are $T$-periodic. From (15), one can see

$$
\begin{aligned}
x_{1}(t+T) & =c_{1} e_{h_{1}}(t+T, 0)=c_{1} e_{h_{1}}(t, 0) e_{h_{1}}(t+T, t) \\
& =c_{1} e_{h_{1}}(t, 0) e_{h_{1}}(T, 0)=c_{1} e_{h_{1}}(t, 0)=x_{1}(t) .
\end{aligned}
$$

Similarly, $y_{1}$ is $T$-periodic. Hence $F\left(\Omega_{x y}\right) \subseteq \Omega_{x y}$. The proof that $F$ is completely continuous is similar to the corresponding work in Theorem 3.1, hence we omit it here. Therefore, by Schauder's fixed point theorem, there exists a fixed point $(x, y) \in \Omega_{x, y}$ such that

$$
(x, y)=F(x, y)=\left(F_{1}(x, y), F_{2}(x, y)\right) .
$$

Now we show that this fixed point is a solution of (1). Let

$$
\begin{aligned}
x(t) & =c_{1} e_{h_{1}}(t, 0)-\int_{t}^{\infty} e_{\ominus h_{1}}(\sigma(u), t) h_{2}(u) y(u) \Delta u \\
& -\int_{t}^{\infty} e_{\ominus h_{1}}(\sigma(u), t) \int_{-\infty}^{u} a(u, s) f(x(s), y(s)) \Delta s \Delta u,
\end{aligned}
$$

and

$$
\begin{aligned}
y(t) & =c_{2} e_{p_{1}}(t, 0)-\int_{t}^{\infty} e_{\ominus p_{1}}(\sigma(u), t) p_{2}(u) x(u) \Delta u \\
& -\int_{t}^{\infty} e_{\ominus p_{1}}(\sigma(u), t) \int_{-\infty}^{u} b(u, s) g(x(s), y(s)) \Delta s \Delta u .
\end{aligned}
$$

Then a delta differentiation with respect to $t$ gives

$$
x^{\triangle}(t)=h_{1}(t) x(t)+h_{2}(t) y(t)+\int_{-\infty}^{t} a(t, s) f(x(s), y(s)) \Delta s,
$$

and

$$
y^{\triangle}(t)=p_{1}(t) x(t)+p_{2}(t) y(t)+\int_{-\infty}^{t} b(t, s) g(x(s), y(s)) \Delta s .
$$

Then $(x, y)$ is a solution of (1). Therefore, $(x, y)$ given by

$$
\begin{aligned}
& x(t)=x_{1}(t)+x_{2}(t), \\
& y(t)=y_{1}(t)+y_{2}(t),
\end{aligned}
$$

is asymptotically $T$-periodic solution of (1).

Acknowledgements. The authors are grateful to the referees for their valuable comments which have led to improvement of the presentation. 


\section{References}

[1] M. Adivar, H.C. Koyuncuoglu, Y.N. Raffoul, Classification of positive solutions of nonlinear systems of Volterra integrodynamic equations on time scales, Commun. Appl. Anal. 16(3) (2012) 359-375.

[2] M. Adivar, Y. N. Raffoul, Existence of periodic solutions in totally nonlinear delay dynamic equations, Electronic Journal of Qualitative Theory of Differential Equations 2009(1) (2009) 1-20.

[3] M. Adivar, Y.N. Raffoul, Existence results for periodic solutions of integro-dynamic equations on time scales, Annali di Matematica 188 (2009) 543-559.

[4] E. Akin, O. Ozturk, On Volterra integro dynamical systems on time scales, Communications in Applied Analysis 23(1) (2019) 21-30.

[5] A. Ardjouni, A. Djoudi, Existence of positive periodic solutions for nonlinear neutral dynamic equations with variable delay on a time scale, Malaya Journal of Matematik 2(1) (2013) 60-67.

[6] A. Ardjouni, A. Djoudi, Existence of periodic solutions for nonlinear neutral dynamic equations with functional delay on a time scale, Acta Univ. Palacki. Olomnc., Fac. rer. nat., Mathematica 52(1) (2013) 5-19.

[7] A. Ardjouni, A. Djoudi, Existence of periodic solutions for nonlinear neutral dynamic equations with variable delay on a time scale, Commun Nonlinear Sci Numer Simulat 17 (2012) 3061-3069.

[8] A. Ardjouni, A. Djoudi, Periodic solutions in totally nonlinear dynamic equations with functional delay on a time scale, Rend. Sem. Mat. Univ. Politec. Torino Vol. 68(4)(2010) 349-359.

[9] M. Bohner, A. Peterson, Dynamic equations on time scales, An introduction with applications, Birkhäuser, Boston, (2001).

[10] M. Bohner, A. Peterson, Advances in dynamic equations on time scales, Birkhäuser, Boston, (2003).

[11] F. Bouchelaghem, A. Ardjouni, A. Djoudi, Existence and stability of positive periodic solutions for delay nonlinear dynamic equations, Nonlinear Studies 25(1) (2018) 191-202.

[12] F. Bouchelaghem, A. Ardjouni, A. Djoudi, Existence of positive solutions of delay dynamic equations, Positivity 21(4) (2017) 1483-1493.

[13] F. Bouchelaghem, A. Ardjouni, A. Djoudi, Existence of positive periodic solutions for delay dynamic equations, Proyecciones (Antofagasta) 36(3) (2017) 449-460.

[14] J.A. Cid, G. Propst, M. Tvrdy, On the pumping effect in a pipe/tank flow configuration with friction, Physica D: Nonlinear Phenomena 273/274 (2014) 28-33.

[15] I. Culakova, L. Hanustiakova, R. Olach, Existence for positive solutions of second-ordre neutral nonlinear differential equations, Applied Mathematics Letters 22 (2009) 1007-1010.

[16] B. Dorociakova, M. Michalkova, R. Olach, M. Saga, Existence and stability of periodic solution related to valveless pumping, Mathematical Problems in Engineering 2018 (2018) 1-8.

[17] M. Gouasmia, A. Ardjouni, A. Djoudi, Periodic and nonnegative periodic solutions of nonlinear neutral dynamic equations on a time scale, International Journal of Analysis and Applications 16(2) (2018) 162-177.

[18] S. Hilger, Ein Masskettenkalkul mit Anwendung auf Zentrumsmanningfaltigkeiten, PhD thesis, Universitat Wurzburg, (1988).

[19] E.R. Kaufmann, Y.N. Raffoul, Periodic solutions for a neutral nonlinear dynamical equation on a time scale, J. Math. Anal. Appl. 319 (2006) 315-325.

[20] V. Lakshmikantham, S. Sivasundaram, B. Kaymarkcalan, Dynamic systems on measure chains, Kluwer Academic Publishers, Dordrecht, (1996).

[21] Z. Li, C. Wang, R.P. Agarwal, D. O'Regan, Commutativity of quaternion-matrix-valued functions and quaternion matrix dynamic equations on time scales, Studies in Applied Mathematics (2020), https://doi.org/10.1111/sapm.12344.

[22] Y.N. Raffoul, Analysis of periodic and asymptotically periodic solutions in nonlinear coupled Volterra integro-differential systems, Turk. J. Math. 42 (2018), 108-120.

[23] D.R. Smart, Fixed points theorems, Cambridge Univ. Press, Cambridge, UK, (1980).

[24] C. Wang, R.P. Agarwal, A classification of time scales and analysis of the general delays on time scales with applications, Mathematical Methods in the Applied Sciences 39(6) (2016) 1568-1590.

[25] C. Wang, R.P. Agarwal, D. O' Regan, R. Sakthivel, Theory of translation closedness for time scales, Developments in Mathematics, Vol. 62, Springer, (2020).

[26] C. Wang, R.P. Agarwal, S. Rathinasamy, Almost periodic oscillations for delay impulsive stochastic Nicholsons blowflies timescale model, Computational and Applied Mathematics 37 (2018) 3005-3026. 